\title{
Simulation Analysis on Determination Technology of Catalytic Discoloring Spectrophotometry of Photometric of Trace Selenium in Marine Organisms by Catalytic Discoloring
}

\author{
1,a Tao Weiran \\ ${ }^{1}$ Tianjin Key Laboratory of Marine Resources and Chemistry, College of Marine Science and \\ Engineering, Tianjin University of Science and Technology, Tianjin 300457, China \\ a,16566345@qq.com
}

\begin{abstract}
Keywords: Marine Organism ; Catalytic Spectrophotometric Determination of Trace; Simulation; Determination of Trace.
\end{abstract}

\begin{abstract}
As the improvement of people's living standard, the content of trace elements in food more and more attract the attention from people. In this paper, I integrate all the analysis situation from Catalytic Kinetic Spectrophotometry to trace elements in the food over the past ten years. Through the NH3-NH4Cl, Se has the inhibitory effect on fading reaction of hydrogen peroxide oxidation G, and develop one new method to stop fading spectrophotometric method and determine trace element Se, the best reaction conditions and the environmental water can be determined by experiments.
\end{abstract}

\section{Introduction}

Catalytic kinetic analysis has been widely used in the quantitative determination of trace elements, the character is high sensitivity $(10 \sim 12 \mathrm{~g} / \mathrm{mL})$, the equipment is simple, so the analysis of trace substances is very often to be seen in in high purity materials, high purity materials, biological samples and environmental samples. The catalytic kinetic spectrophotometric method is Kinetic analysis method which is based on the quantitative relation between the concentration of measurements reaction and reaction rate, taking spectrophotometer, fluorescence spectrophotometer as measurement means. It is different from the traditional thermodynamic methods, the unique of the reaction can measure, and therefore the scope of available chemical reaction can be expanded and the weakness of the traditional photometry can be overcome, making spectrophotometric method a new development. It is not only an important quantitative analysis method, but also one of the important tools to study on the mechanism of catalytic and kinetics reaction[1,5].

Compared with the thermodynamics method, the kinetics method has the following strong points: (1) The selection in the reaction is very good, and is suitable for simultaneous determination in the mixture with properties that are very similar to each other(such as isomers and homologues in organic compound); (2) The Catalytic Kinetic Analysis method has high sensitivity; (3) Expanding the scope of available chemical reaction, because it does not require a complete reaction, only needs the data at the very beginning of the reaction. (4) Because the dynamic analysis method takes the time as variable, which is convenient for the automation of the on-line use of computer and analysis equipments, and is easy to control the process, to determine the sample, to collect and process the data [6-7]. Se is an essential trace element for human beings, and plays an important role on the anti-cancer, anti-aging and other aspects. The main intake of selenium for human is from food, and marine organisms are publically recognized which is enriched if Se. Therefore, the determination of the Se content is very important.

Se is a rare element, the average abundance value in the earth's crust is $0.2 \mathrm{mg} / \mathrm{kg}$, the concentration in natural water is less than $1 \mu \mathrm{g} / \mathrm{L}$. As the continuous mining of antimony, continuous development and widely use of its products, antimony is omnipresent around us, but until now no evidence can approve that antimony is the essential elements for human, however, antimony and its compounds have been recognized as one of the toxic elements by American Environmental Protection Organization (USEPA) and the European Union (EU). The research shows that with different form, the toxicity of antimony is also different, Se (III) as a form of antimony with great toxicity has 
attracted more and more attention form people, therefore, it is important to the establish an effective, rapid, sensitive determination method to determine Se (III) in the environment. At present, the main testing method for $\mathrm{Sb}$ (III) including spectrophotometry, atomic absorption spectrometry, ICP-OES, neutron activation analysis, atomic fluorescence spectrometry, chemical luminescence method, electrochemical method. Compared with above analysis methods, catalytic spectrophotometric method has high sensitivity and good selectivity with simple equipment. So it attracts much attention from people. Especially in recent years, as the new chromogenic agent appears, surface active agent has been used in the solubilization, sensitization of color (fading), which makes the catalytic kinetic spectrophotometric method have a new breakthrough. Because the kinetic spectrophotometric method has high sensitivity, and instrument reagent is easy to have, and the operation is simple and fast, It is widely used in the analysis of trace $\mathrm{Se}$ in recent years [8,9]. Observed from the experiments, Se can significantly catalytic the potassium bromate methyl violet fading reaction in acidic medium, so develop a new method to catalytic kinetic spectrophotometric determine trace Se, and it has been used in the determination Se content in kelp, oysters and other marine biological samples and which has good results.

\section{Experiment}

Instruments and reagents:We choose spectrophotometer with 200 type(Shanghai Unique Instrument Co. Ltd); acidity meter with pHS-3C type (Shanghai Precise Science Instrument Co Ltd); SYZ-B type quartz sub boiling distiller (Jiangsu Qinhua Glass Instrument Factory); FA2004N electronic analytical balance (Shanghai Precision Instrument Factory); SC-501 type super constant temperature water bath (Shanghai Jinping Instrument Company); $\mathrm{Sb}$ (III) standard solution: potassium antimony tartrate $0.2743 \mathrm{~g}$ added into $250 \mathrm{~mL}$ beaker, add into $20 \mathrm{~mL}$ sulfuric acid, heating to dissolve, cooling, moved into $1000 \mathrm{~mL}$ volumetric flask (with $160 \mathrm{~mL}$ (volume ratio of 1: 1) sulfate inside), dilute it with water, shake, it will be standard stock solution with $0.10 \mathrm{mg} / \mathrm{mL}$, dilute to working solution with $5 \mathrm{mu}$ g/mL; $\mathrm{H} 2 \mathrm{O} 2$ solution $(6 \%)$ : put $30 \%$ of $\mathrm{H} 2 \mathrm{O} 250 \mathrm{~mL}$ to into volumetric flask in $250 \mathrm{~mL}$, dilute and constant volume. Solution orange G (OG): 1.0×10-3mol/L; NH3-NH4Cl buffer solution: $\mathrm{pH}=11.05$; all the reagents are analytically pure, and water is two times distilled water.

The Experimental Method:We take two pieces tube in $25 \mathrm{~mL}$, one with some Se inside(catalytic reaction, absorbance is $\mathrm{A}$ ), another without $\mathrm{Se}$ (non-catalytic reaction, absorbance is $\mathrm{A} 0$ ); and then separately add into $3 \mathrm{~mL}$ buffer solution, $1 \mathrm{mLKBrO} 3$ solution and $3 \mathrm{~mL}$ Methyl violet solution, dilute with water, shake, at the same time, put into water in $90 \mathrm{C}$ with continuous temperature for 6 min, quickly take the colorimetric tube out, cooling with running water for $5 \mathrm{~min}$, when it is the same as room temperature, compared with distilled water, at wavelength of $590 \mathrm{~nm}$, measuring A0 and A, calculate the catalytic reaction rate $\Delta \mathrm{A}(\mathrm{A} 0-\mathrm{A})$.

\section{Results and Discussion}

Absorption Curve:The experiment method, measure absorbance A and A0 of inhibitory system and non-inhibitory system in the range of $400 \sim 530 \mathrm{~nm}$, drawing a absorption spectrum, as shown in Figure 1, the maximum absorption wavelength of the two curves were located at $475 \mathrm{~nm}$, no displacement, but the peak increases, we can determine that $\mathrm{Sb}$ (III) has inhibitory effect on $\mathrm{H} 2 \mathrm{O} 2$ oxidation on orange $\mathrm{G}$ fading, so we select $475 \mathrm{~nm}$ as the measuring wavelength. 

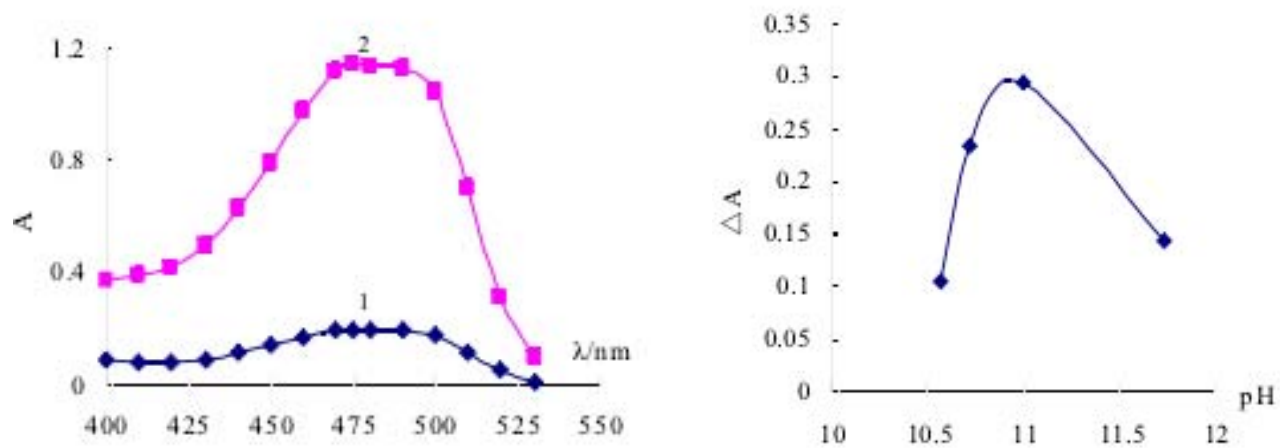

Figure 1 The absorption spectrum of methyl violet

Figure 2 Effect from PH

The Choose of Experiment Conditions: After testing different reaction media, we find that when take the NH3-NH4Cl buffer solution as reaction medium, the system selectivity and sensitivity is better. compared different $\mathrm{NH} 3-\mathrm{NH} 4 \mathrm{Cl}$ buffer solution with different acidity, the experimental results as shown in Figure 2, when $\mathrm{pH}$ is $11.05 \Delta \mathrm{A}$ value is with maximum number, so in the experiment, we choose the $\mathrm{NH} 3-\mathrm{NH} 4 \mathrm{Cl} 11.05$ buffer solution with $\mathrm{pH} 11.05$ to control the acidity of the system.

According to the test method, when test from changing the consumption of $\mathrm{NH} 3-\mathrm{NH} 4 \mathrm{Cl}$ buffer solution of $\mathrm{pH} 11.05$ on sensitivity, the results shown in Figure 3, shows that when the buffer solution concentration is $0.10 \mathrm{~mL}, \Delta \mathrm{A}$ is with maximum value, so in the experiment we use $0.10 \mathrm{~mL}$.

Effect of the Consumption of Orange $\mathbf{G}$ and H2O2:Orange $G$ is as indicators solution in the system, and has impact on the determination of the sensitivity and linear range. The results of the study on the effect of the dosage of sensitivity are shown in Figure 4, with the consumption of orange $\mathrm{G}$ increasing, the value of $\mathrm{A}, \mathrm{A} 0$ and $\Delta \mathrm{A}$ are also increasing constantly, sensitivity of the system has been increased, but the absorbance is too big, and the accuracy decreased, in order to ensure the $\mathrm{A}$ value is around 1 , we choose orange $\mathrm{G}$ in $1.80 \mathrm{~mL}$.

In this experiment we choose $\mathrm{H} 2 \mathrm{O} 2$ as the oxidant, according to the test method, we make the test with $6 \% \mathrm{H} 2 \mathrm{O} 2$ (Figure 5), the results show that with the increase of the consumption, the $\Delta \mathrm{A}$ is also increasing and when it is around $4.00 \sim 6.00 \mathrm{~mL}, \Delta \mathrm{A}$ is with the maximum value and stable, so in the experiment, we choose $5.00 \mathrm{~mL}$.
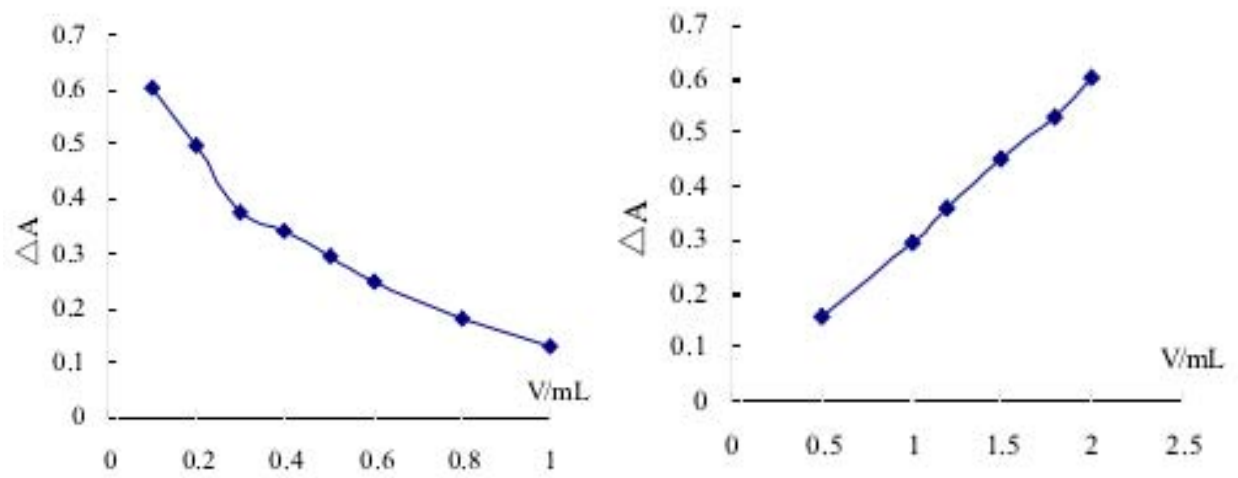

Figure 3 Influence of dosage of NH3-NH4Cl Figure 4 Effect from the indicator

Effect from Reaction Temperature:Fixing the other conditions during the experiment process, only change the reaction temperature, determining of $\Delta \mathrm{A}$ value under different temperature, the results are shown in Figure 6, when the temperature is lower than $50 \mathrm{C}$, the fading reaction of inhibition system and non-inhibitory system are not obvious, so we can use running water to cool and stop the reaction. When the temperature is higher than $50 \mathrm{C}$, with the increasing of the temperature Delta $\Delta \mathrm{A}$ value is gradually increasing, in order to get a higher sensitivity and convenient to operate, we choose boiling water to heat and running water to stop the reaction. 


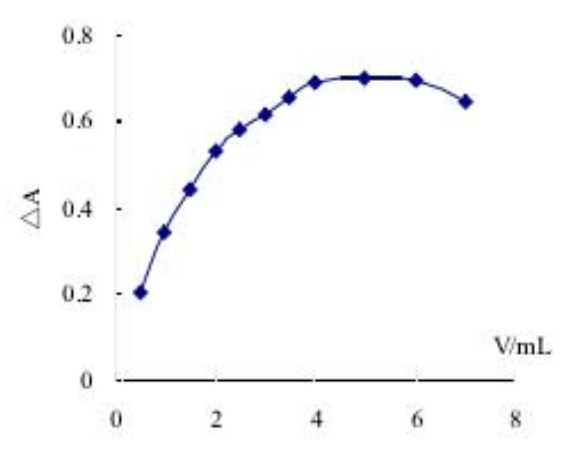

Figure 5 Effect form oxidant

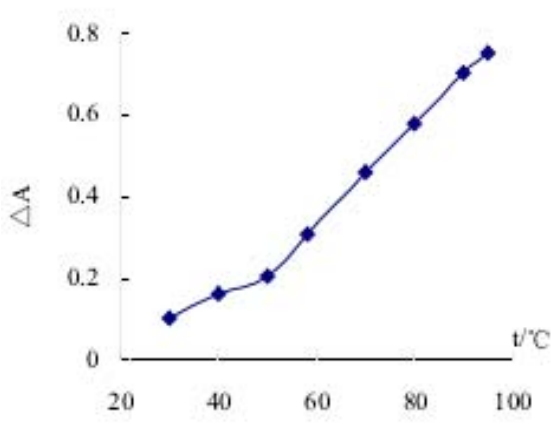

Figure 6 Effect from reaction temperature

Working Curves and Detection Limits of the Method:Under the selected experiment conditions, when the the concentration of Se is in $0 \sim 8.0 \mu \mathrm{g} \mathrm{L}$ and it has good linear relationship with $\Delta \mathrm{A}$, the regression equation was: $\Delta \mathrm{A}=0.0069 \mathrm{Cse}(\mu \mathrm{g} \mathrm{L})+0.0075(\mathrm{r}=0.9983)$. According to $\mathrm{b}=1 \mathrm{~cm}$, $\Delta \mathrm{A}=0.001$ [2], we can get that this method for the detection limit of Se is $0.14 \mu \mathrm{g} \mathrm{L}$, equivalent to fluorescence spectrophotometry of DAN.

Influence and Elimination of Coexisting Ions:The determination of $0.1 \mu \mathrm{g}$ Se also tests some effect from some common ions. The results showed that: when the relative error is within $\pm 5 \%, 5000$ times of $\mathrm{K}+, \mathrm{Na}+, \mathrm{Ca} 2+, \mathrm{NO}-3, \mathrm{Cl}-, 2000$ times of $\mathrm{Mg} 2+, \mathrm{Zn} 2+, \mathrm{Ni} 2+, \mathrm{SO} 2-4,1000$ times of $\mathrm{Al3}+$, $\mathrm{Co} 2+, \mathrm{Ti}, \mathrm{Mo}, \mathrm{Se}, 200$ times of Mn2+,100 times of NO-2, I- and 10 times of Fe3+, Cu2+ does not influence the determination. When take the natural sea water instead of distilled water in the experiments, we further analyzes the comprehensive effect of coexisting ions. Table 1 show that the high concentration of $\mathrm{Fe} 3+, \mathrm{Mn} 2+$ and $\mathrm{Cu} 2+$ in the marine environment has serious interference on the experiment. In the $6 \mathrm{~mol} \mathrm{LHCl}$ medium, when methyl isobutyl ketone (MIBK) has been extracted, the recovery rate is $96.8 \%$, shows that the influence from coexisting components are effectively eliminated.

Table 1 Effect from sea water to the determination of Se

\begin{tabular}{|l|l|l|l|}
\hline System & Distilled water & Sea water & MIBK extracted sea water \\
\hline$\triangle \mathrm{A}$ & $0.062,0.067,0.063$ & $-0.070,-0.055,-0.078$ & $0.064,0.061,0.058$ \\
& $(0.064,4.1 \%)$ & $(-0.068 \%, 17 \%)$ & $(0.061,4.9 \%)$ \\
\hline
\end{tabular}

Analysis on the Samples: Water samples needs to be filtered quickly by the filter paper, to remove the insoluble impurities, absorbed filtrate needs to be separated by cation exchange resin to separate and remove $\mathrm{Co} 2+, \mathrm{Cu} 2+, \mathrm{Fe} 3+$ and other cation, take exchange liquid $30 \mathrm{~mL}$, and add into $6 \mathrm{~mol} / \mathrm{L}$ with $3 \mathrm{~mL}, 10 \%$ sulfate with $4.5 \mathrm{~mL}$, heating in a boiling water for $30 \mathrm{~min}$, reduce the $\mathrm{Sb}(\mathrm{V})$ in water to $\mathrm{Sb}$ (III), after cooling, use deionized water to constant volume to $50 \mathrm{~mL}$. Get processed solution with $10 \mathrm{~mL}$, measuring according to experiment method, and do the recovery experiments at the same time, results are shown in table 2.

Table 2 analytical results of sample antimony (III) and recoveries rate $(\mathrm{n}=6)$

\begin{tabular}{|l|l|l|l|l|l|l|}
\hline Samples & $\begin{array}{l}\text { Determination } \\
\text { value of water } \\
(\mu \mathrm{g} / \mathrm{mL})\end{array}$ & $\begin{array}{l}\text { Determination } \\
\text { value } \\
\text { solution }(\mu \mathrm{g} / 10 \mathrm{~mL})\end{array}$ & $\begin{array}{l}\text { Added } \\
\text { scalar } \\
(\mu \mathrm{g} / 10 \mathrm{~mL})\end{array}$ & $\begin{array}{l}\text { Measurement } \\
\text { results } \\
(\mu \mathrm{g} / 10 \mathrm{~mL})\end{array}$ & $\begin{array}{l}\text { Recovery } \\
\text { rate }(\%)\end{array}$ & $\begin{array}{l}\text { RSD } \\
(\%)\end{array}$ \\
\hline $\begin{array}{l}\text { Spring } \\
\text { water }\end{array}$ & 0.0250 & 0.150 & 1.0 & 1.109 & 95.9 & 3.4 \\
\hline $\begin{array}{l}\text { River } \\
\text { water }\end{array}$ & 0.0541 & 0.324 & 1.0 & 1.302 & 97.8 & 2.5 \\
\hline
\end{tabular}

Processing of Biological Samples Before the Treatment: Experiments show that, under selected conditions, Se can catalyzed potassium bromate oxidation of methyl violet fading, other forms of Se do not have this function. Therefore, to determine the total Se in biological samples, we need to separate them at the same time, transfer all the form into Se. Wet digestion is a commonly used decomposition method. Considering the recovery rate and sensitivity, mixed acid HNO3-HClO4 is a perfect digestion solution, and only can transfer the organic Se and Se0 oxidize to Se and Se will not 
change. Therefore, the sample digested by mixed acid $\mathrm{HNO} 3-\mathrm{HClO} 4$, still need to further oxidize to Se.

This paper we choose to soaked samples with nitric acid for the whole night, when most organic matter has been decomposed, add into mixed acid HNO3-HClO4 (4: 1) with low temperature $(<200 \mathrm{C})$ to heat and decompose. Test organic Se with containing $0.1 \mathrm{~g}$ Se according to this, the recovery rate is in $95.2 \% \sim 99.7 \%$, shows a complete conversion, and no volatilization loss. In order to simplify the operation,in this paper we adopts the same medium with MIBK extraction and purification of digestion solution. Experiment shows that: in $6 \mathrm{~mol} \mathrm{LHCl} \mathrm{medium,} \mathrm{use} \mathrm{boiling} \mathrm{water} \mathrm{to} \mathrm{heat} \mathrm{for} 10$ min, the conversion rate is $96 \%$; Heating above $30 \mathrm{~min}$, Se will have a loss, so in this paper we choose a boiling water for $20 \mathrm{~min}$.

\section{Conclusion}

Inbuffer solution of NH3-NH4Cl medium, Se has the inhibitory effect of on hydrogen peroxide oxidation orange $\mathrm{G}$ fading reaction, and establish a new method to determinate Fading Spectrophotometric method of trace Se, the best reaction conditions are determined by experiments, and the environmental water samples were determined.

\section{References}

[1] Lai Haitao, Bai Yue. Using Methylene Blue Catalytic Kinetic Spectrophotometric for the Determination of Trace Selenium in foods (IV) [J]. Food and Fermentation Industry, 2006,32 (2): 102-105.

[2] Dong Binggang, Liu $\mathrm{Yu}$, Sang Demin. Determination of Trace Selenium in food by Spectrophotometry [J]. Trace Elements and Health Research, 2006,23 (4): 43-46. 2006,23 (4): 43-46. [3] Ni Xiuzhen, Wang Xiaoju. Determination of Trace Iodine by Sixteen Alkyl Bromide Catalytic Kinetic Spectrophotometry Method in Food [J]. Food Science and Technology, 2008,33 (5): 226-228.

[4] Zhang Dong, Yu Ping, Zhao Guangsheng. Determination of Ttrace Two Aniline by Inhibitory Catalytic Kinetic Photometry [J]. Physical and Chemical Inspection (chemical), 2007, 43 (8): 680-681.

[5] Chen Yahong, Wu Zhihao, Luo Danming, B Determination of Hemoglobin Bleaching [J]. Chemical Reagent, fading method 2007, 29 (3): 151-153

[6] Song Xuesheng. Orcein Fading Spectrophotometric method for the determination of trace copper [J]. Metallurgical Analysis, 2006, 26 (3): 62-64.

[7] Chen Yujing. The Determination of Trace Cr(VI) of Catalytic Zinc Enrichment Separation of Corn by Glutenenrichment Catalytic Spectrophotometric Method [J]. Analysis Laboratory, 2006, 25 (2): 66-68

[8] Fang Xia. Determination of Trace Formaldehyde in beverage by Catalytic kinetics of potassium bromate - azurol I photometric system [J]. Food and Science, 2009,30 (6): 199-202.

[9] Cai Xiangwen. Determination of Trace Copper in Food by spectrophotometry [J]. Guangzhou chemical, 2009,37 (4): 141-144..

[10] Wang Hongfu, Determination of Trace Chromium Cr (VI) -H2O2-4,5- Dibromo Fluorescein System for Catalytic Spectrophotometric Method, [J]. Metallurgy 2007, 27 (6): 55-57 